\title{
Incidencia de factores psicosociales en la educación superior en la carrera de medicina de la Escuela Superior Politécnica de Chimborazo
}

\author{
Incidence of Psychosocial Factors in Higher Education in the Career of \\ Medicine of the Polytechnic School of Chimborazo
}

Silvana Paola Ocaña Coello ${ }^{1}$, Ángel Floresmilo Parreño Urquizo ${ }^{2}$, Martha Cecilia Bonilla Caicedo $^{3}$, Azucena Amparo Ocaña Coello ${ }^{4}$

\begin{abstract}
.
The educational teaching process encompasses intellectual processes, psychological resources where students can face problems of daily life. Numerous research shows evidence of the influence of personal and environmental factors on the teaching-learning process. Conditioning factors are studied such as: the level of knowledge, motivational level for the subject, the future profession and the learning strategies used among others. But in the learning environment, intrinsic personal factors are involved and a certain value is not assigned to these hidden problems in the person. This research addresses psychosocial problems of ESPOCH Medicine students and their influence on academic performance,

${ }^{1}$ Escuela Superior Politécnica de Chimborazo, Facultad de Salud Pública. Riobamba, Ecuador. socana@espoch.edu.ec

${ }^{2}$ Escuela Superior Politécnica de Chimborazo, Facultad de Ciencias. Riobamba, Ecuador. aparreno@espoch.edu.ec

${ }^{3}$ Escuela Superior Politécnica de Chimborazo, Facultad de Ciencias. Riobamba, Ecuador. martha.bonilla@espoch.edu.ec

${ }^{4}$ Escuela Superior Politécnica de Chimborazo, Facultad de Ciencias. Riobamba, Ecuador. am.par.24@hotmail.com
\end{abstract}


emphasizes the importance of addressing social problems that compromise the integrity of young people.

Key Words: Psychosocial Risks, Assertiveness, Vulnerability, Social phobia/ /Academic performance

\section{Resumen.}

El proceso docente educativo abarca procesos intelectuales, recursos psicológicos donde los estudiantes puedan enfrentar problemas de la vida cotidiana. Numerosas investigaciones muestran evidencias de la influencia de factores personales y ambientales sobre el proceso enseñanza aprendizaje. Se estudian factores condicionantes como: el nivel de conocimientos, nivel motivacional por la asignatura, la futura profesión y las estrategias de aprendizaje empleadas entre otras. Pero en el entorno del aprendizaje intervienen factores personales intrínsecos y no se asigna un valor determinado a estos problemas ocultos en la persona. Esta investigación aborda problemáticas psicosociales de los estudiantes de la carrera de Medicina ESPOCH y su influencia en el rendimiento académico, enfatiza la importancia de abordar problemas sociales que comprometen la integridad de los jóvenes.

Palabras clave: Riesgos Psicosociales, Asertividad, Vulnerabilidad, Fobia social, Rendimiento académico

\section{Introducción.}

Los factores de riesgo psicosociales en la actualidad (Mangaoang, 2013) son más frecuentes que los registrados en los medios de comunicación y de investigación, por su carga psicoafectiva en el entorno social, siendo ésta una problemática poco abordada, el interés de ahondar es el descubrimiento de la magnitud y la presencia de los mismos en el entorno universitario.

Numerosas investigaciones (Yeimi, 2011) (Jiménez, 2010)muestran evidencias de la influencia de múltiples factores de índole personal y ambiental (George, J, 2013) sobre el proceso de enseñanza aprendizaje. Algunos factores condicionantes del aprendizaje que se puede mencionar el nivel de partida o conocimientos previos, el nivel motivacional por la asignatura y la futura profesión, las estrategias de aprendizaje empleadas entre otras. 
Pero en el entorno del proceso de aprendizaje, también intervienen factores personales que son intrínsecos (Jiménez, 2010) y que pocas veces se asigna un valor determinado a esta serie de problemas ocultos en la persona y que demandan de una intervención urgente, ya que ciertas problemáticas desencadenan trastornos emocionales severos y que nacen de condicionantes sociales que también necesitan ser estudiadas para comprender su influencia y su comportamiento.

La investigación aborda estas problemáticas psicosociales desde los estudiantes de primero a tercer semestre de la carrera de medicina de la ESPOCH y la influencia en su rendimiento académico (Satpathy, S, 2012), entendiendo que resulta un esfuerzo magno el poder estudiar todas las problemáticas sociales en un mismo entorno en un período de tiempo definido, por lo que, se priorizan tres problemas psicosociales considerados importantes potenciales de trastornos emocionales en la población estudiantil: trastorno de ansiedad social o fobia social, la vulnerabilidad social y la asertividad.

El estudio es netamente descriptivo, interpretativo, correlacional, para comprender el fenómeno, además se realiza un corte de tipo transversal (Sampiere, 2010) en el tiempo, la intensión del mismo es apoyar el desarrollo global de las capacidades de jóvenes que acuden a la carrera de medicina en la ESPOCH, en una de las áreas más complicadas actualmente (Yeimi, 2011) por la dinámica social, para lo cual, se hace un análisis sistemático del problema con el propósito de describir, interpretar y entender su naturaleza.

Se parte de datos que son recogidos en forma directa, mediante la aplicación de test psicológicos que miden e interpretan cada uno de los problemas psicosociales investigados, además se fundamenta con datos de fuentes secundarias (actas de calificaciones semestrales obtenidas en la secretaría de la Escuela de Medicina de la Facultad de Salud Pública y su cuerpo colegiado), para completar el estudio. (Feldman, 2010)

La cobertura analizada y la factibilidad de hacerlo define trabajar con el total de la población de estudio que son 228 casos, considerando algunas condicionantes que son relevantes antes del desarrollo de la misma, como es el número de estudiantes que por cualquier razón solamente asisten a tres o menos cátedras por paralelo, número de estudiantes que por 
cualquier razón no asisten de manera regular o no están presentes en el momento de la aplicación de los instrumentos, y el número de estudiantes que disponen de matrícula pero han desertado por cualquier razón.

El trabajo permitió descubrir que el $15.25 \%$ de estudiantes padece de trastorno de ansiedad social, afectando mayoritariamente a las mujeres en un $73.5 \%$; un dato interesante el $27.8 \%$ tienen un alto riesgo de desarrollar esta patología; en referencia a la vulnerabilidad psicosocial se encontró que el $71.3 \%$ de estudiantes la padece y de este porcentaje el $62 \%$ son mujeres considerando a este grupo como más vulnerable debido a las diversas responsabilidades en lo familiar, laboral y social; con respecto a la asertividad un $33.95 \%$ de estudiantes tienden a la no asertividad que pueden desencadenar en otros trastornos como la ansiedad, el estrés, la fobia social entre otros. La influencia de estos factores con el rendimiento académico según el estudio realizado concluye que con un promedio de notas aproximado de 17 puntos sobre 20 al parecer no tienen mayor predominio en su preparación estudiantil superior.

\section{Metodología.}

La presente investigación se ha considerado el uso de varias metodologías descriptivas utilizando tres instrumentos específicos, el primero que permitió determinar el Trastorno de Ansiedad Social, con el test de Inventario de Fobia Social (SPIN). La Fobia Social se valoró en cada uno de los participantes de la investigación, con 17 preguntas puntuables entre 0 y 4 puntos, siendo por tanto las puntuaciones mínima y máxima posibles de 0 y 68 respectivamente; estas puntuaciones medias obtenidas en estos experimentos (con desviación estándar) han sido Población normal 12,1 ( \pm 9,3) y Población clínica 41,1 ( \pm 10,2). (Anastasi, 2010)

El segundo instrumento permitió valorar el grado la Vulnerabilidad psicosocial, fue identificado con un test de 20 preguntas puntuables entre 1 y 5 puntos (puntuaciones mínima y máxima posibles de 20 y 100 respectivamente). Los autores del test han establecido una escala de valoración por cada criterio posible de respuesta (Casi siempre $=1$, Muchas veces $=2$, Algunas veces $=3$, Pocas veces $=4$ y Casi nunca $=5$ ). Los puntos de corte según el autor, se establece como una media entre 40 puntos, lo que significa que el puntaje del test 
que en suma se encuentre en menos de 40 puntos es considerado que no existe vulnerabilidad psicosocial y se lo llama "normal", mientras que si la puntuación es igual o mayor a 40 puntos significa que existe una vulnerabilidad psicosocial llamada "no adecuado". (De la Fuente, 2012)

El tercer instrumento para realizar la investigación del asertividad en el grupo de estudio, se usa el test de Lazarus comparando los totales asertivos y no asertivos, determinando el predominio de cada uno de ellos indicando la tendencia asertiva o no asertiva.

Un cuarto insumo es el registro del rendimiento académico de cada estudiante de los semestres en estudio, insumo que se registra desde la secretaría de la Escuela de Medicina.

Dada la importancia de la investigación se toma en cuenta a todo el universo de 285 estudiantes de primero a tercer semestre bajo condicionantes, el número de estudiantes que por cualquier razón solamente asisten a tres o menos cátedras por paralelo, número de estudiantes que por cualquier razón no asisten de manera regular o no están presentes en el momento de la aplicación de los instrumentos, y el número de estudiantes que disponen de matrícula pero han desertado por cualquier razón, quedando con un total de 228 estudiantes. (Fernández, 2004)

El proceso de investigación se realiza en fases o períodos de actividades siendo las siguientes: Fase Preliminar: La investigación se prepara y planifica, se diseña un perfil de proyecto y se hace una proyección del alcance y la sostenibilidad de la misma.

Fase I: Recoger información preliminar sobre los problemas psicosociales a investigar, revisión de bibliografía, análisis de instrumentos disponibles.

Fase II: Aplicación de los instrumentos en los grupos objetivos y ejecución de la planificación. A la par, las actividades de recolección van acompañadas del análisis de contexto, es decir, de las posibilidades de encontrar o entender las causas que desencadenan los problemas psicosociales. 
Fase III: Tabulación, ordenamiento y análisis de la información recogida, rescatar la información más relevante para medir los indicadores de la investigación, por tanto, selección de información relevante para responder a los objetivos de la investigación.

Fase IV: Compilación de toda la información preliminar y la de los resultados obtenidos, para ordenar el documento de presentación.

\section{Resultados y discusión.}

La población objetivo del estudio está definida por todos los estudiantes matriculados y que asisten regularmente a la carrera de Medicina de la Facultad de Salud Pública de la ESPOCH, en los semestres de primero a tercero, los hallazgos en este grupo en particular se presentan según sexo, según edad y según procedencia. (Feuerstein, 1980)

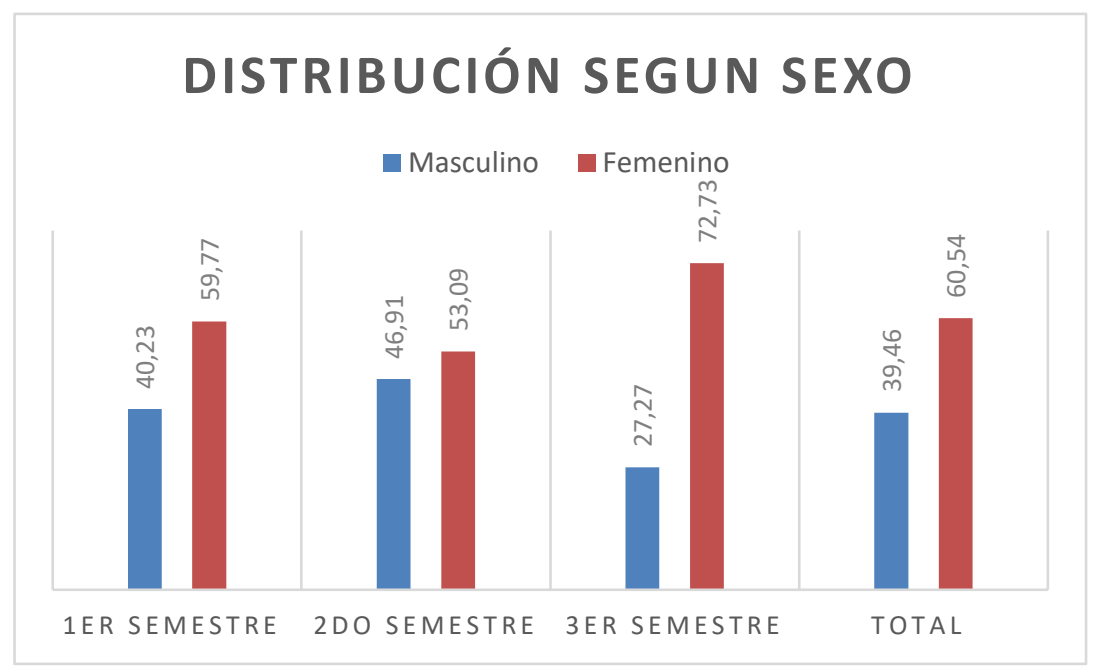

Gráfico No. 1 Distribución del grupo de investigación según sexo

Se observa cierta paridad en cuanto a la distribución por sexo solamente en el primero y segundo semestre, aunque no es simétrica, en el primer caso la superioridad del sexo femenino es notoria $60 \%$ a un $40 \%$, en el segundo caso la brecha se acorta de un $53 \%$ a un 47\%. Llama la atención en el tercer semestre, en donde la brecha se incrementa significativamente de $73 \%$ al $27 \%$, este fenómeno puede deberse a diferentes condiciones que no se abordan en esta investigación pero que denota una preocupación a ser investigada sobre la deserción o disminución del número de hombres en el avance de la carrera, es decir, conocer que pasa en semestres superiores y cuál es el comportamiento de la presencia de estudiantes según sexo. (Fortul, Varela, \& Ávila, 2010). 
Sin embargo, el hallazgo en la investigación muestra que de cada 10 estudiantes 6 son mujeres en la carrera de Medicina.

Por tanto, el estudio, considera para su análisis esta relación porcentual entre sexo femenino que es del $60 \%$, frente al del sexo masculino que es del $40 \%$. En los análisis se hará un breve recordatorio de esta diferencia, especialmente de los problemas psicosociales por sexo.

Otro de los datos que son relevantes para la investigación es conocer las edades del grupo objetivo, los problemas psicosociales, afectan a la mayoría de la población, pero se enfoca en circunstancias que son exclusivamente nuevas que el ser humano debe afrontar y, que se presentan de manera gradual a lo largo de la vida. En la niñez, la adolescencia, la juventud ocurren diferentes cambios psicológicos que dependen del entorno y de las condiciones sociales que enfrenta, como el estudio, el trabajo, el deporte, las citas, etc. (Coll, Palacios, Marchesi, 1992) En el grupo de estudio se encontró un 82,5 \% con edades comprendidas entre los 18 a 20 años, siendo su mayor concentración los 19 años con el 36,8\%, seguido de la edad de 20 años con el 23,3\% y finalmente el 22,4\% para la edad de 18 años.

Por otro lado, se identificó que los jóvenes proceden de 19 provincias del Ecuador, actualmente y desde la constitución del año 2008 en el país existen 24 provincias, la cobertura de la carrera actualmente beneficia al 79\% de las provincias del Ecuador. Otro hallazgo interesante en este análisis es mencionar que la provincia de Chimborazo es la que tiene mayor presencia en esta carrera, con un predominio del sexo femenino. Este hecho se repite en las demás provincias, es decir, la carrera de medicina tiene un predominio del sexo femenino, aun cuando provienen de diferentes provincias. Tungurahua, Pichincha e Imbabura son las provincias de mayor demanda en orden de afluencia de estudiantes a la carrera. (Freud, 2010)

\section{Situación de jóvenes de la carrera de medicina con relación a la Fobia Social}

La fobia social es un trastorno de ansiedad que entre sus características presenta una intensa timidez, ansiedad y persistentes a enfrentar determinadas personas, así como miedo a las reuniones sociales, interacción con los demás o simplemente a ser observados en cualquier 
situación, hasta tal punto que lo dicho anteriormente influye directamente en la vida diaria de quien la padece. (George, J, 2013)

De los 223 estudiantes encuestados, se encontró que el 15,25 \% de ellos son pacientes clínicos que padecen el trastorno de ansiedad social o fobia social, lo cual, se puede representar con la relación de que en la carrera de medicina de la ESPOCH de cada 10 estudiantes 2 padecen fobia social. Dato estadístico relevante para la toma de decisiones, además de mencionar que 3 de cada 10 se encuentran en riesgo de padecer el trastorno, lo que en suma se plantea que 4 de cada 10 estudiantes tienen o están en riegos de tener el trastorno de ansiedad social. (Vygotsky, 1988)

Además, el 73,5 \% de los estudiantes que padecen el trastorno son de sexo femenino, el $26,5 \%$ son hombres, lo cual, se puede interpretar que el problema afecta mayoritariamente a las mujeres, en una relación de 7 a 3 por cada 10 estudiantes.

TABLA No. 1 Fobia Social en Estudiantes de Medicina de la ESPOCH FOBIA SOCIAL EN ESTUDIANTES DE MEDICINA - ESPOCH

\begin{tabular}{|c|c|c|c|c|c|c|c|c|}
\hline & \multicolumn{3}{|c|}{$\mathbf{N}^{\circ}$} & \multirow[b]{2}{*}{ Total } & \multicolumn{3}{|c|}{$\%$} & \multirow{2}{*}{$\begin{array}{c}\% \\
\text { Total }\end{array}$} \\
\hline & $\begin{array}{c}\text { 1er } \\
\text { Semestre }\end{array}$ & $\begin{array}{c}\text { 2do } \\
\text { Semestre }\end{array}$ & $\begin{array}{c}\text { 3er } \\
\text { Semestre }\end{array}$ & & $\begin{array}{c}\text { 1er } \\
\text { Semestre }\end{array}$ & \begin{tabular}{|c|}
$2 d o$ \\
Semestre
\end{tabular} & $\begin{array}{c}\text { 3er } \\
\text { Semestre }\end{array}$ & \\
\hline Normal & 49 & 43 & 35 & 127 & 21,97 & 19,28 & 15,70 & 56,95 \\
\hline Normal al limite & 25 & 24 & 13 & 62 & 11,21 & 10,76 & 5,83 & 27,80 \\
\hline Clínico & 12 & 14 & 7 & 33 & 5,38 & 6,28 & 3,14 & 14,80 \\
\hline Clínico Severo & 1 & 0 & 0 & 1 & 0,45 & 0,00 & 0,00 & 0,45 \\
\hline total & 87 & 81 & 55 & 223 & 39,01 & 36,32 & 24,66 & 100,00 \\
\hline
\end{tabular}

Situación de jóvenes de la carrera de medicina con relación a la vulnerabilidad psicosocial 


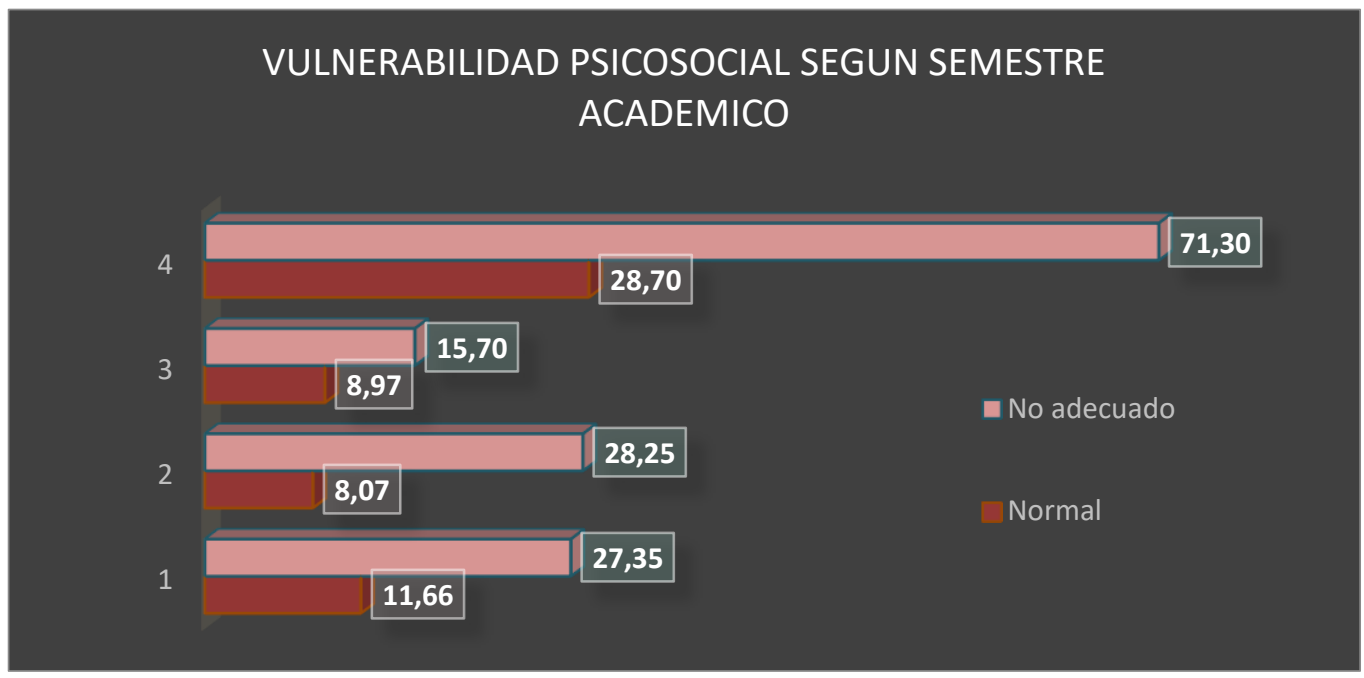

Gráfico No. 2 Vulnerabilidad Psicosocial

En este gráfico se aprecia una visualización de la globalidad del problema, es decir, 7 de cada 10 estudiantes presenta vulnerabilidad psicosocial. (Martínez, 2010).

Esta estadística debe considerarse para la toma de decisiones en cómo puede abordarse esta problemática desde el rol de la universidad y de la responsabilidad que tiene en el bienestar de los estudiantes.

\section{VULNERABILIDAD PSICOSOCIAL Y SU RELACION ENTRE SEXOS}

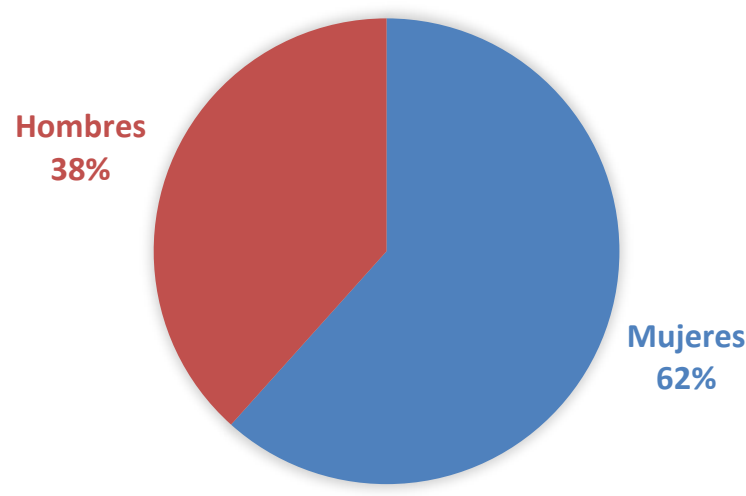

Gráfico 3 Vulnerabilidad Psicosocial y su Relación entre Sexo 
El problema de vulnerabilidad psicosocial afecta mayoritariamente al grupo de mujeres (62 $\%$ ) frente al de hombres (38\%). (Valladares, 2010)

Los propios procesos naturales asociados a su naturaleza cíclica y reproductiva, la mayor exposición a situaciones que generan un alto grado de sufrimiento (violencia de género, pobreza, pobreza extrema) entre otros indicadores sociales. (Osorio, s/f)

Según la procedencia de los estudiantes que presentan vulnerabilidad psicosocial, se puede ver que las provincias de Cotopaxi y Tungurahua son las que presentan mayor tendencia, en tercer lugar, esta Imbabura y Chimborazo en cuarto lugar. (Ortiz, 2009).

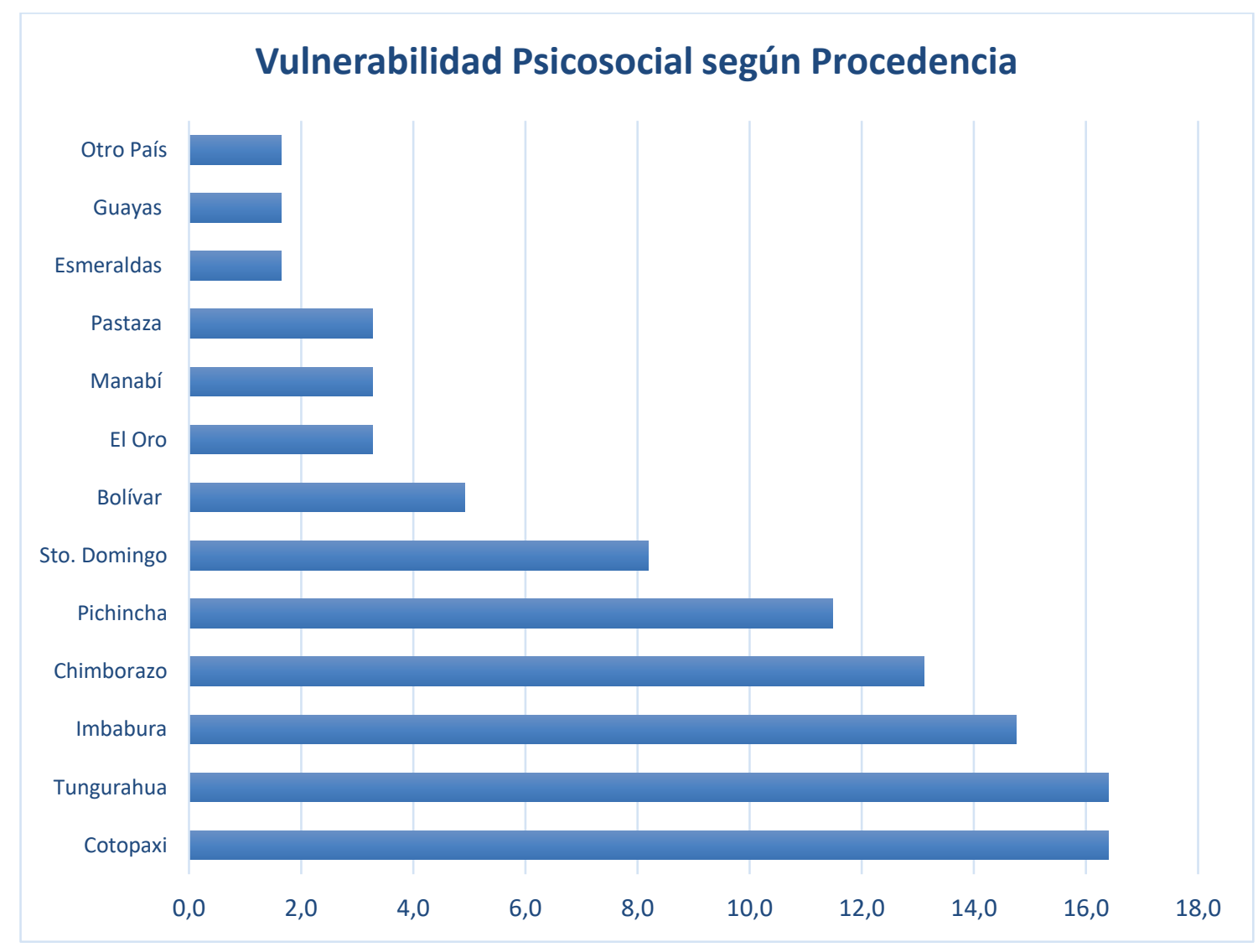

Gráfico 4 Vulnerabilidad Psicosocial Según la Procedencia 
Situación de jóvenes de la carrera de medicina con relación al asertividad

Tabla No. 2 Asertividad en Estudiantes de Medicina

\section{ASERTIVIDAD EN ESTUDIANTES DE MEDICINA - ESPOCH}

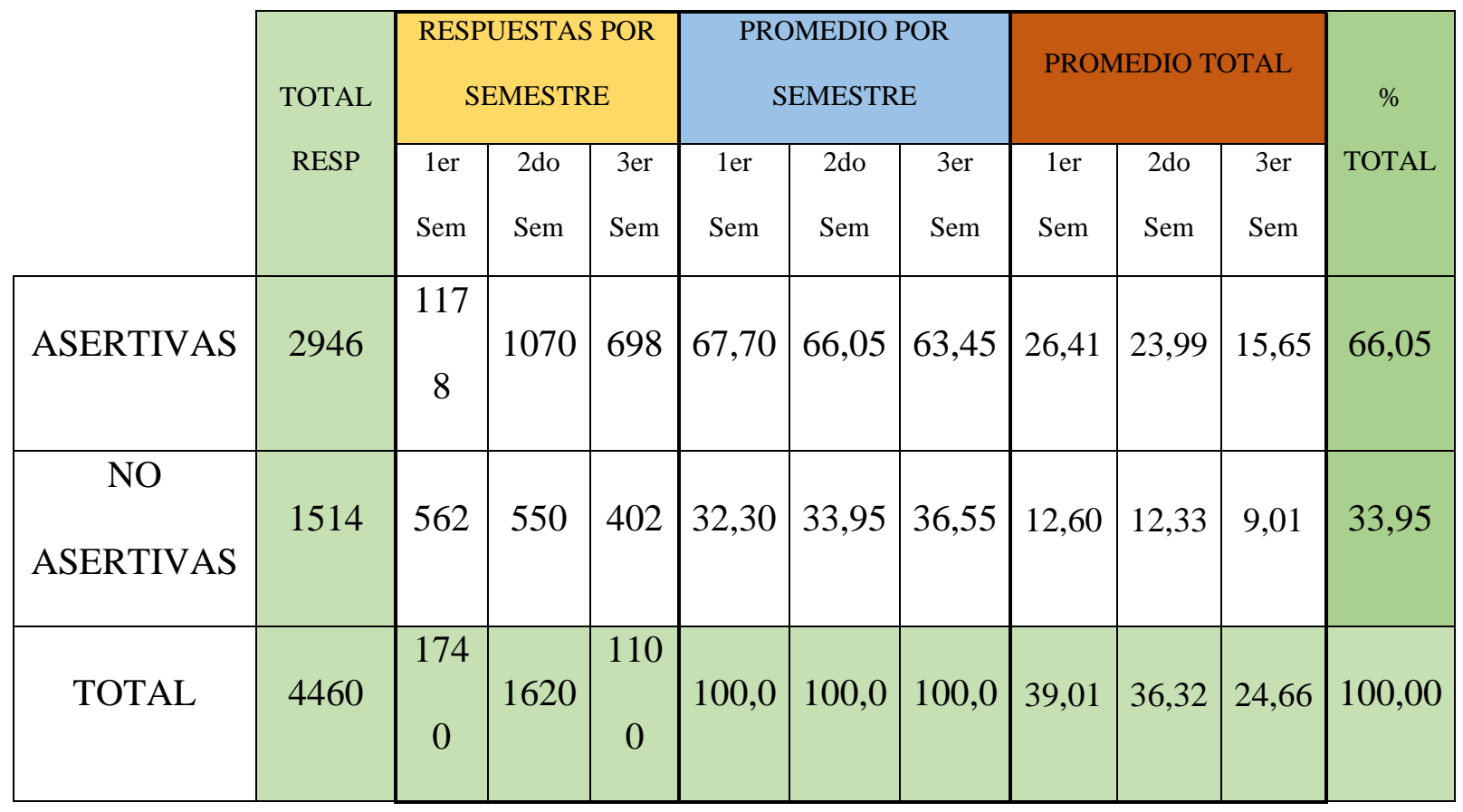

De manera general para la investigación, los resultados de la aplicación del test de Lazarus a los estudiantes del estudio tornan un escenario similar a los hallazgos descritos por semestre, el $66,05 \%$ de los estudiantes son asertivos y el $33,95 \%$ no lo son.

El problema es de la minoría, sin embargo, si analizamos desde la relación de que por cada 10 estudiantes 3 tienen una tendencia no asertiva, el problema se lo grafica como imperioso de ayuda y de intervención focalizada. (Papalia, D, 2010) 
La conducta asertiva se funda en el respeto: el respeto a uno mismo, a los demás y al sistema de valores de cada uno Con estos hallazgos en la investigación estaríamos asegurando que 3 estudiantes de cada 10 no defienden sus derechos, no defiende sus intereses o necesidades, por tanto, se tornan en un grupo por demás vulnerable y con el riesgo de padecer otros tipos de trastornos como la ansiedad, el estrés, la fobia social, entre otras.

\section{Relación entre los problemas psicosociales y el rendimiento académico}

El rendimiento académico es una medida de las capacidades del estudiante, que expresa lo que éste ha aprendido a lo largo del proceso formativo. También supone la capacidad del alumno para responder a los estímulos educativos. En este sentido, el rendimiento académico está vinculado a la aptitud.

Existen distintos factores que inciden en el rendimiento académico. Desde la dificultad propia de algunas asignaturas, hasta la gran cantidad de exámenes que pueden coincidir en una fecha, pasando por la amplia extensión de ciertos programas educativos, son muchos los motivos que pueden llevar a un alumno a mostrar un pobre rendimiento académico. (Ausubel, 1983).

Otras cuestiones están directamente relacionadas al factor psicológico, como la poca motivación, el desinterés o las distracciones en clase, que dificultan la comprensión de los conocimientos impartidos por el docente y termina afectando al rendimiento académico a la hora de las evaluaciones. Entendiendo que las causas que originan estas distracciones dependen de factores que están en torno a la vida del estudiante. (Salanova, 2003)

Para el estudio que ahora se presenta, las condiciones de rendimiento académico son muy alentadoras, pese a haber demostrado que existen problemas psicosociales que afectan un gran porcentaje de estudiantes de la carrera de medicina. (Sandler, 2010).

Para el grupo de estudiantes del primer semestre - que fueron considerados según se describe en la metodología de esta investigación- existe un rendimiento grupal de 17,75 puntos sobre 20 en el sexo masculino, y de 17,71 puntos sobre 20 en el sexo femenino. Lo cual no perece verse afectado por los problemas psicosociales estudiados. 
Tabla 3 Rendimiento Académico Según el Sexo

\begin{tabular}{ccc}
\hline \multicolumn{3}{c}{ RENDIMIENTO } \\
ACADEMICO SEGÚN SEXO \\
\hline Sexo & Total & Promedio \\
masculino & 42 & 17,75 \\
femenino & 54 & 17,71 \\
& 96 & \\
\hline
\end{tabular}

De acuerdo al análisis los problemas no inciden de manera significativa en el rendimiento académico, sin embargo, el estudio, hace referencia a las problemáticas individuales, en donde se pueden evidenciar que los estudiantes que padecen algún problema psicosocial descrito, no forma parte de un grupo social, es decir, el rendimiento académico bueno que tienen, se debe a su aislamiento social y a su separación de las condiciones sociales necesarias para el desarrollo integral de una persona. (Carrion, 2010).

En el caso del grupo de segundo semestre, se puede notar una baja en el rendimiento académico por parte de las mujeres en relación a los hombres, puede deberse a la afectación de algún problema psicosocial encontrado, y en donde se pudo ratificar que las mujeres son mayormente más vulnerables a este tipo de trastornos. (Willcox, 2011).

Sin embargo, el promedio del grupo de mujeres es 16,89 puntos sobre 20, que no es una mala evaluación, pero que si llama la atención por ser un grupo vulnerable, es decir, algo pasa en su rendimiento y en su desarrollo integral. 
Tabla 4 Rendimiento Académico Según el Sexo

\begin{tabular}{ccc}
\hline Sexo & Total & Promedio \\
\hline Femenino & 43 & 16,89 \\
Masculinos & 33 & 17,22 \\
& 76 & \\
\hline
\end{tabular}

Este grupo de personas que padecen algún problema psicosocial se excluye del sistema social, lo que causa aislamiento social, y dedicación exclusiva a una actividad que puede resultarle satisfactoria o necesaria para sus aspiraciones, el estudiar, pero de forma individual, si contactos colectivos, sin las necesidades de buscar ayuda y apoyarse en sus pares. La pregunta obvia es qué va a pasar con este tipo de futuros profesionales en el área de medicina que deben interactuar con la sociedad, dada su responsabilidad frente a la sociedad, si no tienen control sobre su estima, sobre sus temores o miedos, sobre su facilidad de relacionamiento, cómo podrán desarrollar sus actividades profesionales en el futuro. (Coll, 2010)

TABLA No. 5 Rendimiento Académico Según el Sexo

\begin{tabular}{ccc}
\hline Sexo & Total & Promedio \\
\hline Femenino & 17 & 16,24 \\
Masculinos & 39 & 17,38
\end{tabular}

56

El grupo del tercer semestre muestra una tendencia igual al grupo de segundo, el rendimiento académico es más bajo en mujeres que en hombres, aunque no sea mayormente una diferencia abrumadora, las condiciones en las que se encuentran estos hallazgos, hacen pensar que efectivamente existe un grado de influencia de los problemas psicosociales con el rendimiento académico. (Zabalza, 2010) 
Se evidencia que el impacto de padecer un trastorno psicosocial es mayor en las mujeres, por tanto, existe un problema social que merece una atención por parte de la institucionalidad que tiene la responsabilidad de asumir grandes retos para apoyar el proceso de formación de los estudiantes que asisten a esta institución. (Tejedor, 2010)

\section{Conclusiones:}

- Existe Fobia Social, 2 de cada 10 estudiantes la padece, y 3 de cada 10 están en riesgo alto de desarrollar el trastorno.

- El trastorno de ansiedad social afecta mayoritariamente a las mujeres, por cada 10 estudiantes que padecen el trastorno 7 son mujeres.

- Promocionar la atención global, a través de programas de sensibilización para toda la población, programas asistenciales para colectivos de alto riesgo y formación para profesionales de la salud, la educación y la atención social, podría ser parte de un proceso de atención hacia los estudiantes de las diferentes carreras.

- Existe vulnerabilidad psicosocial en el $70 \%$ de estudiantes de la carrera de medicina por cualquier causa, esto depende de factores externos e internos.

- La vulnerabilidad afecta mayoritariamente a las mujeres en relación de 6 a 4 por cada 10 estudiantes que está expuesto.

- El asertividad es una condición de seguridad y estima, el no ser asertivo genera un problema, éste afecta a la minoría, sin embargo, la relación supone urgente intervención ya que por cada 10 estudiantes 3 tienen una tendencia no asertiva.

- Existe afectación de los problemas psicosociales en el rendimiento académico de los estudiantes, sin embargo, no son exclusivos.

- La afectación en el rendimiento académico es mayor para las mujeres, definidas por sus condiciones propias de género.

- Integrar acciones para el colectivo de más alto riesgo, incorporando la perspectiva de género y la sensibilización de profesionales de la atención a las personas, para dinamizar la prevención y la promoción de hábitos de salud.

\section{Referencias Bibliográficas.}

Anastasi, A. (2010). Tests Psicológicos. México: Pretince Hall.

Ausubel, D. (1983). Psicología Educativa: Un punto de vista cognoscitivo. México: Trillas.

Carrion, S. (2010). Programación Neuro Linguística. Colombia: CREDIMAR.

Coll, M. (2010). Desarrollo Psicológico y Educación . Madrid: Alianza. 
Coll, Palacios, Marchesi. (1992). Desarrollo Psicológico y Educación. Madrid: Alianza - 2da Edición.

De la Fuente, J. (2012). Salud Mental y Medicina Psicológica . México: McGraw-Hill.

Feldman, R. (2010). Psicología: con aplicaciones en países de habla hispana. México: McGraw-Hill Interamericana.

Fernández, P. (2004). Validity and reliability of the spanish modified versión of the trait meta-mood scale. Psychological Reports, 751-755.

Feuerstein, R. (1980). “Instrumental Enrichment”.

Fortul, T., Varela, T., \& Ávila, M. (2010). Factores que influyen en los estilos de aprendizaje en los estudiantes de medicina. Revista de Educación Superior, 138.

Freud, S. (2010). Introducción al Psicoanálisis. Buenos Aires: Alianza.

George, J. (2013). Perceptions of tutora and student nurses on factors that influence academic performance at a nursing college. Sudafrica: University of the Free State.

Jiménez, B. M. (2010). Factores de riesgos psicosociales, formas consecuencias y buenas prácticas. Madrid: Universidad Autónoma de Madrid.

Managaoang, N. (2013). Factors influencing the academic performance in physics of dmmmmsu-mluc laboratory high school. Fourth year students. International Scientific Research Journal, 2-5.

Martínez, E. (2010). Las Psicoterapias Existenciales. Bogotá: Manual Moderno.

Ortiz, A. (2009). Manual para elaborar el modelo pedagógico de la institución escolar. Madrid: Antillas.

Osorio, R. (s/f). Aprendizaje y Desarrollo - Vygotsky. Obtenido de http://www.nodo50.org/sindpitagoras/Vigosthky.htm 
Papalia, D. (2010). Psicología del Desarrollo. Bogotá: McGraw-Hill Interamericana.

Papalia, D., \& Feldman, R. (2012). Desarrollo Humano. México: McGraw Hill.

AzuSampiere, R. H. (2010). Metodologia de la Investigación. México: Interamericana editores S.A .

Sandler, J. (2010). Psicopatología Teoría sdel Aprendizaje Ivestigación y Aplicaciones . Mexico: Trillas.

Satpathy, S. (2012). Psychosocial and demographic correlates of academic performance of hearing-impaired adolescents. Asia Pacific Disability Rehabilitation Journals, 19.

Tejedor, F. (2010). Los alumnos de la Universiadad de Salamanca: características y redimiento académico. Salamanca: Ediciones Universidad de Salamanca.

Valladares, I. (2010). Psicología del Aprendizaje. Loja: Universidad Particular de Loja.

Vygotsky, L. (1988). El Desarrollo de los Procesos Psicológicos Superiores. Mexico: Grijalbo.

Willcox, M. (2011). Factores de riesgo y protección para el rendimiento académico. Revista Iberoamericana de Educación, 55.

Zabalza, M. (2010). La enseñanza universitaria. El escenario y sus protagonistas . España: Narcea.

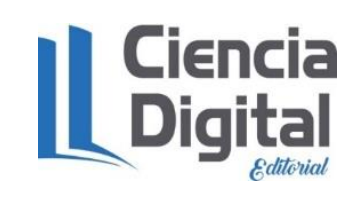




\section{Para citar el artículo indexado.}

Ocaña Coello, S., Parreño Urquizo, Ángel, Bonilla Caicedo, M., \& Ocaña Coello, A. (2019). Incidencia de factores psicosociales en la educación superior en la carrera de medicina de la Escuela Superior Politécnica de Chimborazo. Explorador Digital, 3(3.1), 59-76. https://doi.org/10.33262/exploradordigital.v3i3.1.865

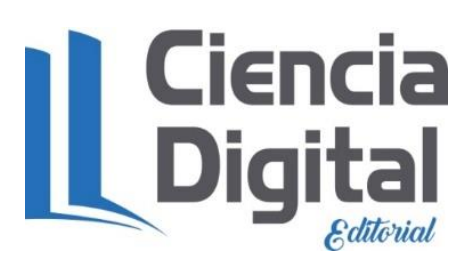

El artículo que se publica es de exclusiva responsabilidad de los autores y no necesariamente reflejan el pensamiento de la Revista Explorador Digital.

El articulo queda en propiedad de la revista y, por tanto, su publicación parcial y/o total en otro medio tiene que ser autorizado por el director o editor de la Revista Explorador

Digital.
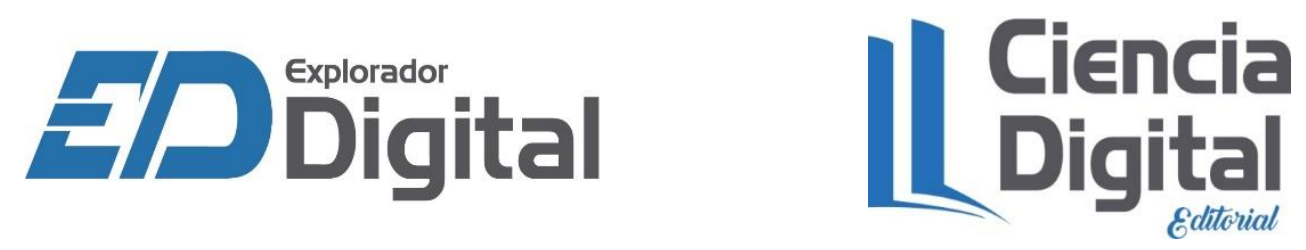